\title{
Qualitative and Numerical Research of Body Motion in a Resisting Medium
}

\author{
MAXIM V. SHAMOLIN \\ Lomonosov Moscow State University \\ Institute of Mechanics \\ Michurinskii Ave., 1, 119192 Moscow \\ RUSSIAN FEDERATION
}

\begin{abstract}
If in $[1,2]$, we represent the asymptotical stability conditions of the rectilinear translational deceleration (drag), and in $[2,3]$, we obtained the new multiparametric family of phase patterns in the space of quasi-velocities, then in this activity, we prepare the qualitative material for the preparation of further natural experiments on the motion of the hollow circular cylinders in a medium.
\end{abstract}

Abstract: Proposed activity presents next stage of the study of the problem of the plane-parallel motion of a rigid body interacting with a resistant medium through the frontal plane part of its external surface. Under constructing of the force acting of medium, we use the information on the properties of medium streamline $\mathrm{fl} \mathrm{w}$ around in quasistationarity conditions (for instance, on the homogeneous circular cylinder input into the water). The medium motion is not studied, and we consider such problem in which the characteristic time of the body motion with respect to its center of masses is comparable with the characteristic time of motion of the center of masses itself.

Key-Words: Rigid body, Resisting Medium, Qualitative and numerical Analysis, Experiment Received: March 10, 2021. Revised: July 19, 2021. Accepted: August 1, 2021. Published: August 6, 2021.

\section{Preliminaries}

Let give the brief summary to the previous stages of studying. Also, by the reason of complexity of nonlinear analysis, the initial stage of such a study is the neglecting of the dependence of the medium interaction force moment on the angular velocity and use of such dependence on the angle of attack only [1,3]).

From the practical view point it is important the problem of studying of stability of so-called unperturbed (rectilinear translational) motion under which the velocities of body points are perpendicular to the plate (cavitator).

The whole spectrum of results found under the simplest assumption on the absence of the medium damping action on a rigid body allowed the author to make the conclusion that it is impossible to fin those conditions under which there exist the solutions corresponding to the angular body oscillations of a finit amplitude.

The experiment in the motion of homogeneous circular cylinders in the water (see $[4,5]$ ) justifie that in modelling the medium action on the rigid body, it is also necessary to take account of an dependence of the medium interaction force moment on the angular velocity of the body. Herewith, there arise the additional members that brings a dissipation to the system.

In studying the class of body motions with the finit angles of attack, the principal problem is find ing those conditions under which there exist the finit amplitude oscillations in a neighborhood of the unperturbed motion. Therefore, there arises the necessity of a complete nonlinear study.

In earlier author's works, one has succeeded to use the instability of the rectilinear translational body motion for the methodological purposes (see [5]), i. e., in determination of unknown parameters of the medium action on the body in quasi-stationarity conditions.

The account of the medium damping action on the rigid body leads to an affirmat ve answer to the principal question of the nonlinear analysis: under the body motion in a medium with finit angles of attack, in principle, there can arise stable auto-oscillations which can be explained by the account of an additional dependence of the medium action on the body angular velocity that brings an additional dissipation to the system.

Furthermore, under the applying of methodology of studying of the dissipative dynamical systems of certain type, we obtain the new multi-parametric family of phase patterns on the two-dimesional cylinder; this family consists of the infinit set of topologically 
non-equivalent phase patterns changing its topological types under the variation of the system parameters by the degenerate way (see also [6]).

\section{Introduction}

The presented work is the study of the problem of a rigid body motion interacting with a medium through frontal plane part (the plate of its external surface). Under constructing of the force acting of medium, we use the information on the properties of medium streamline $\mathrm{fl} \mathrm{w}$ around in quasi-stationarity conditions $[7,8]$. The medium motion is not studied, and we consider such problem in which the characteristic time of the body motion with respect to its center of masses is comparable with the characteristic time of motion of the center of masses itself.

By the reason of complexity of nonlinear analysis, the initial stage of such a study is the neglecting of the dependence of the medium interaction force moment on the angular velocity and use of such dependence on the angle of attack only (see also [8]).

From the practical view point it is important the problem of studying of stability of so-called unperturbed (rectilinear translational) motion under which the velocities of body points are perpendicular to the plate (cavitator).

The whole spectrum of results found under the simplest assumption on the absence of the medium damping action on a rigid body allows the author to make the conclusion that it is impossible to fin those conditions under which there exist the solutions corresponding to the angular body oscillations of a finit amplitude.

The experiment in the motion of homogeneous circular cylinders in the water (see [9]) justifie that in modelling the medium action on the rigid body, it is also necessary to take account of an dependence of the medium interaction force moment on the angular velocity of the body. Herewith, there arise the additional members that brings a dissipation to the system.

As mentioned above, in studying the class of body motions with the finit angles of attack, the principal problem is findin those conditions under which there exist the finit amplitude oscillations in a neighborhood of the unperturbed motion. Therefore, there arises the necessity of a complete nonlinear study.

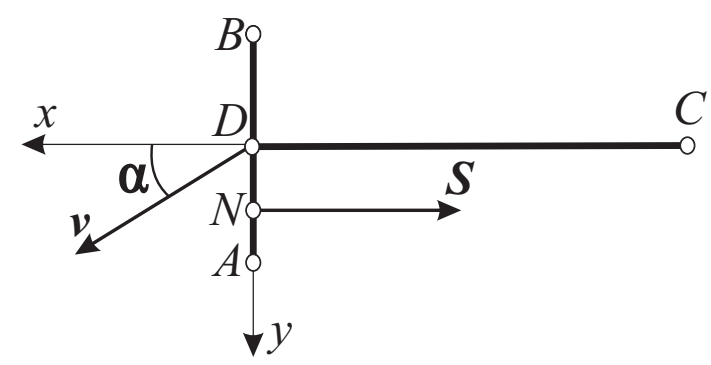

Figure 1: Plane-parallel motion of symmetric rigid body in a resisting medium

\section{Plane-Parallel Motion of Symmet- ric Rigid Body in a Resisting Medium}

Assume that a homogeneous rigid body of mass $m$ executes a plane-parallel motion in a uniform $\mathrm{fl} \mathrm{w}$ of medium, and that a certain part of the external body surface is a plane plate being under the medium streamline $\mathrm{fl} \mathrm{w}$ around conditions. In the case of absence of tangent forces, this means that the action of the medium on the plate reduces to the force $\mathbf{S}$ (applied at the point $N$ ) whose line of action is orthogonal to the plate (Fig. 1). Let the remained part of the body surface be situated in a volume bounded by the fl w surface that goes away from the plate boundary and is not subjected by the medium action. For example, similar conditions can arise after the body entrance into the water [9]. We also assume that gravity force acting to the body, is negligible small in contrast with the resistance (interaction) force of a medium.

Let us relate to the body the right coordinate system $D x y z$ whose axis $z$ moves parallel to itself, and for simplicity, assume that the plane $D z x$ is the geometric symmetry plane of the body. Then

among the possible motions, there exists the regime of the rectilinear translational drag (the unperturbed motion) which is perpendicular to the plate $A B$. Herewith, the perpendicular dropped from the body center of gravity $C$ on the plate plane belongs to the line of the action of the force $\mathbf{S}$. And under the perturbation of this regime, the velocity vector $\mathbf{v}$ of the point $D$ relatively the medium, deviates, in general, from the axis $D C$ of geometric symmetry on the certain angle (of attack) $\alpha$.

To construct the dynamical model, let us introduce the firs three phase coordinates: the value $v$ of the velocity of the point $D$ (Fig. 1), the angle $\alpha$, and the algebraic value $\Omega$ of the projection of the body absolute angular velocity on the axis $z, A B=\Delta$.

Assume that the value of the force $\mathbf{S}$ quadrati- 
cally depends on $v S=s_{1} v^{2}$ with certain coeffi cient $s_{1}$ (Newtonian drag). One usually represents $s_{1}$ in the form $s_{1}=\rho P c_{x} / 2$, where $c_{x}$ is now the dimension-free coefficien of the frontal resistance ( $\rho$ is the medium density and $P$ is the plate area). This coefficien depends on the angle of attack, the Struchal number, and other quantities which are usually considered as parameters in the static models. In what follows, we also introduce the following additional phase variable of the "Struchal type" $\omega \cong \Omega \Delta / v$, and also the auxiliary function $s=s_{1} \operatorname{sgn} \cos \alpha$, herewith, the interaction of a medium to the body is define by the pair of functions $\left(y_{N}, s\right)$.

Let us defin the dependence of coefficien $s_{1}$ on the angle of attack, i. e., in principle, we assume that the value $s$ is the function of $\alpha$, and the value $y_{N}=D N$ is the function of the pair of dimensionfree variables $(\alpha, \omega)$.

As was mentioned, the previous works (see, for instance, $[8,10])$ are devoted to such studying of plane-parallel interaction of the body with a medium, in which we take into account the dependence of the pair $\left(y_{N}, s\right)$ on the angle of attack only. We also study the plane-parallel and spatial body motions in nonlinear statement in the case of dependence of the value $s$ on the angle of attack, and under the condition of an additional dependence of the function $y_{N}$ on the normalized angular velocity $\omega$.

The problem of a free deceleration of the body with the small angles of attack forms the further notation on the nonlinear dynamical systems describing the interaction of a medium with the body taking account of so-called rotational derivatives of the moment with respect to the body angular velocity. The term "rotational derivative" is often used in hydrodynamics in the case when the differentiability of dynamical functions makes in non-inertial coordinate system; furthermore, if the moment of force depends on the angular velocity then in is present in the linear form on the angular velocity and in the motion equations. ties

The unperturbed motion is define by the equali-

$$
\alpha(t) \equiv 0, \omega(t) \equiv 0 .
$$

Therefore, we use the function $y_{N}(\alpha, \omega)$ for small $(\alpha, \omega)$ in the form

$$
y_{N}=\Delta(k \alpha-h \omega),
$$

where $k$ and $h$ are the certain constants. Because the geometrical symmetry of the body (which ensuring evenness of function $s$ ), we can ignore the dependence of $s$ on $\alpha$.

The linearized model of the force medium action contains three parameters $s=s_{1}, k, h$, which are de- termined by the plate form in the plan. The firs of these parameters, the coefficien $s$, is dimensional. The parameters $k$ and $h$ are dimension-free because of the method of their introduction.

Note that the quantities $s$ and $k$ can be found experimentally by using weight measurements in devices of the hydro- or aerodynamic tubes type. In [11], there is also the information about the theoretical find ing of these quantities for separate plate forms. This information allows us to assume that $k>0$. As for the parameter $h$ (which introduces into the system the dependence of the moment with respect to the body angular velocity), even the very necessity of its introduction to the model is not a priori obvious.

To study the properties of the motion of the classes of bodies considered, in Institute of Mechanics of M. V. Lomonosov Moscow State University (see $[11,12])$, one carried out the experiments in registration of the motion of homogeneous circular cylinders in the water. The experiment allows one to make several important conclusions.

First: the rectilinear stationary free body drag (the unperturbed motion in the water) is unstable at least with respect to the angle of attack and the angular velocity. Owing to the experiment, it becomes possible to fin the dimension-free parameters $k$ and $h$ of the medium action on a rigid body.

The second of them is as follows: in modelling the medium action on the body, it is necessary to take account of the additional parameter characterizing the rotational derivative of the moment of the hydro- or aerodynamical forces with respect to the body angular velocity. This parameter introduces the dissipation into the system.

In certain cases, the value of the damping moment coefficien under the body motion in the water was already estimated in [13]. This estimate confirm the instability of the body rectilinear motion in the water. Purely formally, increasing the value of the damping coefficient we can attain the stability of a motion, but it is difficul to ensure this stability in reality. The rigid body rectilinear motion is stable in certain media (for example in the clay), as the experiment shows. Possibly, this stability is attained owing to the existence of a considerable damping from the medium in the system or for the existence of forces tangent to the plate.

Note that the unperturbed motion may also be called rectilinear translational deceleration.

The position of the body on the plane is specifie by the coordinates $\left(x_{0}, y_{0}\right)$ of the point $D$ and by an angle $\varphi$. The polar coordinates $(v, \alpha)$ of the tip of the velocity vector of the point $D$ and the algebraic value of the projection of the angular velocity $\Omega$ are related to the variables $\left(\dot{x_{0}}, \dot{y_{0}}, \dot{\varphi}, \varphi\right)$ by the nonintegrable re- 
lations:

$$
\dot{\varphi}=\Omega, \dot{x_{0}}=v \cos (\alpha+\varphi), \dot{y_{0}}=v \sin (\alpha+\varphi) .
$$

Thus, the phase state of the system is determined by the functions

$$
\left(v, \alpha, \Omega, x_{0}, y_{0}, \varphi\right)
$$

and the firs three quantities being considered quasivelocities.

Since the kinetic energy of the body and the generalized forces do not depend on its position on the plane, the coordinates $\left(x_{0}, y_{0}, \varphi\right)$ are cyclic, which reduces the order of the system of equations of motion.

The equations of motion of the center of mass (for the projections onto the body axes $D x y$ ) and the angular momentum equations in the König frame form a closed system of differential equations in the threedimensional phase space of quasi-velocities $(\sigma=D C$ and $I$ is the central moment of inertia; differentiation is with respect to time)

$$
\begin{gathered}
\dot{v} \cos \alpha-\dot{\alpha} v \sin \alpha-\Omega v \sin \alpha+\sigma \Omega^{2}=-\frac{s(\alpha) v^{2}}{m}, \\
\dot{v} \sin \alpha+\dot{\alpha} v \cos \alpha+\Omega v \cos \alpha-\sigma \dot{\Omega}=0 \\
I \dot{\Omega}=y_{N}(\alpha, \omega) s(\alpha) v^{2}, \omega \cong \frac{\Delta \Omega}{v} .
\end{gathered}
$$

Systems (3), (4)-(6) form a complete system of equations to describe the quasistationary planeparallel motion of a rigid body in a resisting medium.

\section{Medium Interaction Functions De- pendency on Angular Velocity of the Body}

The dynamic system (4)-(6) includes the functions $y_{N}(\alpha, \omega)$ and $s(\alpha)$, which defin the action of the medium on the body. The function $y_{N}$ (compare with (2)), except of angle of attack $\alpha$, depends on the reduced angular velocity $\omega$. In particular, if we neglect the latter dependence on the angular velocity (which is the so-called elementary suggestion on the medium interaction functions), then $y_{N}$ is a function of the angle of attack only: $y_{N}=y(\alpha)$, and its dependence on the unique argument can be determined by using the experimental information about the properties of the streamline fl w around [11]. Further, in this casef, we can apply the method of "submersion" of the problem in a more general class of problems.

But the objective of this section is the account of such an influenc for the rotational derivatives of the moment with respect to the body angular velocity components. For this account, it takes the introducing in the medium interaction functions the additional arguments, which is a nontrivial modeling problem, in principle. As already mentioned, in this work we introduce the angular velocity as an argument into the function $y_{N}$ only, and neglect of this introducing in reduced coefficien $s$.

By analogy with (2), we consider the value $y_{N}$ in the following form:

$$
y_{N}(\alpha, \omega) \cong y_{N}(\alpha, \Omega / v)=y(\alpha)-\frac{H \Omega}{v},
$$

furthermore, by the results of experiment (see [5]), $H>0$.

Then the Eq. (6) has the following form:

$$
I \dot{\Omega}=F(\alpha) v^{2}-H s(\alpha) \Omega v, F(\alpha)=y(\alpha) s(\alpha) \text {. }
$$

The system (4), (5), (8) contains the functions $F(\alpha), s(\alpha)$, the explicit forms of which we are not able to describe analytically even for the bodies of a simple form. Up to the present, because of complexity, we use the method of "submersion" of this problem in a more general class of problems which takes into account the qualitative properties of the functions $F(\alpha), s(\alpha)$ only.

The S. A. Chaplygin' result (in studying the plane-parallel $\mathrm{fl} \mathrm{w}$ around of a plane plate of infinit length by a homogeneous medium $\mathrm{fl} \mathrm{w}$, see $[5,6]$ ) at findin the medium interaction functions $y(\alpha)$ and $s(\alpha)$ analytically, is the reference result for us:

$$
\begin{gathered}
y(\alpha)=A \sin \alpha \in\{y\}, A=y^{\prime}(0)>0, \\
s(\alpha)=B \cos \alpha \in\{s\}, B=s(0)>0 .
\end{gathered}
$$

The result of S. A. Chaplygin helps us to construct the functional classes $\{y\}$ and $\{s\}$. Combining Eqs. (9) and (10) with the experimental information about the streamline fl w around properties [7], we describe formally the given classes. They consist of sufficientl smooth $2 \pi$-periodic $(y(\alpha)$ is odd and $s(\alpha)$ is even) functions satisfying the following conditions: $y(\alpha)>$ 0 for $\alpha \in(0, \pi)$, and, moreover,

$$
y^{\prime}(0)>0, y^{\prime}(\pi)<0
$$

(the function class $\{y\}=Y$ );s( $\alpha)>0$ for $\alpha \in$ $(0, \pi / 2), s(\alpha)<0$ for $\alpha \in(\pi / 2, \pi)$, and, moreover,

$$
s(0)>0, s^{\prime}(\pi / 2)<0
$$

(the function class $\{s\}=\Sigma$ ). Both $y$ and $s$ change the sign under the replacement of $\alpha$ on $\alpha+\pi$. Therefore,

$$
y \in Y, s \in \Sigma \text {. }
$$


In what follows, there rises the product

It follows from the conditions listed above that function $F$ (introduced in (8)) is a sufficientl smooth odd $\pi$-periodic function satisfying the following conditions: $F(\alpha)>0$ for $\alpha \in(0, \pi / 2)$,

$$
F^{\prime}(0)>0, F^{\prime}(\pi / 2)<0
$$

(the function class $\{F\}=\Phi$ ).

In particular, the analytic function

$$
\begin{gathered}
F=F_{0}(\alpha)=A B \sin \alpha \cos \alpha \in \Phi, \\
A B=y^{\prime}(0) s(0),
\end{gathered}
$$

is also a typical representative of the function class $\Phi$ arisen.

In connection with the noted in [9] instability of the perturbed motion, we can arise the following question: are whether there exist the (bounded) finite amplitude angular oscillations of the body's axis of symmetry?

Let formulate the more general question: is whether there exists a pair of functions $y$ and $s$ such that equation $0<\alpha(t)<\alpha^{*}<\pi / 2$ holds for certain solution of the dynamical part of the motion equations beginning from the certain moment $t=t_{1}$ ?

As shown in [11], under the elementary restriction on the functions $y_{N}$ and $s$, we have shown that for a quasi-static description of the interaction between the medium and the body, when the dynamical quantities $y_{N}$ and $s$ depend only on the angle of attack, for any admissible pair of obtained dynamical functions $y$ and $s$, in the whole range of finit angles of attack $(0<\alpha<\pi / 2)$, there are no any the (bounded) finite amplitude angular oscillations in the system considered.

Thus, to attain a possible affirmat ve answer to the principal question (arising above) of nonlinear analysis, in modelling the interaction of a body with a medium, we take into account an additional dependence of the moment on the reduced body angular velocity, therefore, we use the Eq. (7) for $H>0$. Furthermore, under certain conditions, we expect a possible positive answer to this principal question.

Certainly, only the analysis of the equations of motion in the neighborhood of unperturbed motion is of practical importance since the lateral surface is wetted at some critical angles of attack, which makes our model invalid. And for bodies with different lateral surfaces, however, the critical angles are generally different and unknown. Therefore, the entire range of angles has to be examined.

Thus, to study a plane-parallel $\mathrm{fl} \mathrm{w}$ past a plate, use is made of classes of dynamic systems define in terms of a pair of medium interaction functions, which considerably complicates the qualitative analysis.
In principle, the approach considered in this paper can be partially combined with the finit element method.

\section{Free Deceleration of a Rigid Body in a Resisting Medium}

Similar to the choice of medium interaction functions, we defin the dynamic functions $s$ and $y_{N}$ in the system (4)-(6) in the form (7), (13). Therefore, in the system the additional damping (but in the certain domains of the phase space and dispersing) moment of a nonconservative force is present in considered system as before.

As before, introducing new dimensionless phase variable and differentiation by the formulas

$$
\Omega=n_{0} v \omega, \quad<\cdot>=n_{0} v<^{\prime}>,
$$

system (4)-(6) is reduced to the following form:

$$
\begin{gathered}
v^{\prime}=v \Psi(\alpha, \omega), \\
\alpha^{\prime}=-\omega+\mu_{2} \omega^{2} \sin \alpha+\frac{\mu_{2}}{I n_{0}^{2}} F(\alpha) \cos \alpha- \\
-\frac{\mu_{2}}{I n_{0}} H \omega s(\alpha) \cos \alpha+\frac{s(\alpha)}{m n_{0}} \sin \alpha \\
\omega^{\prime}=\frac{F(\alpha)}{I n_{0}^{2}}+\mu_{2} \omega^{3} \cos \alpha-\frac{\mu_{2}}{I n_{0}^{2}} \omega F(\alpha) \sin \alpha- \\
-\frac{H}{I n_{0}} \omega s(\alpha)+\frac{\mu_{2}}{I n_{0}} H \omega^{2} s(\alpha) \sin \alpha+\frac{s(\alpha)}{m n_{0}} \omega \cos \alpha, \\
\Psi(\alpha, \omega)=-\mu_{2} \omega^{2} \cos \alpha+\frac{\mu_{2}}{I n_{0}^{2}} F(\alpha) \sin \alpha- \\
-\frac{\mu_{2} H}{I n_{0}} \omega s(\alpha) \sin \alpha-\frac{s(\alpha)}{m n_{0}} \cos \alpha,
\end{gathered}
$$

herewith, we defin the dimensionless parameters $\mu_{1}, b=\mu_{2}, H_{1}=\mu_{3}$ as follows:

$$
\begin{gathered}
\mu_{1}=2 \frac{B}{m n_{0}}, b=\sigma n_{0}, H_{1}=\frac{B H}{I n_{0}}, \\
n_{0}^{2}=\frac{A B}{I} .
\end{gathered}
$$

Two latter equations (18) of system (17), (18) form the independent second-order subsystem on the phase cylinder $\mathbf{S}^{1}\{\alpha \bmod 2 \pi\} \times \mathbf{R}^{1}\{\omega\}$.

As before, we study the stability problem of trivial solution of system (18), obviously, corresponding to the rectilinear translational deceleration unperturbed motion.

To this end, we write the corresponding characteristic equation near the origin of coordinates:

$$
\begin{gathered}
\lambda^{2}-\lambda\left[\mu_{1}+\mu_{2}-\mu_{3}\right]+ \\
+\frac{\mu_{1}}{2}\left(\frac{\mu_{1}}{2}+\mu_{2}-\mu_{3}\right)+1=0 .
\end{gathered}
$$




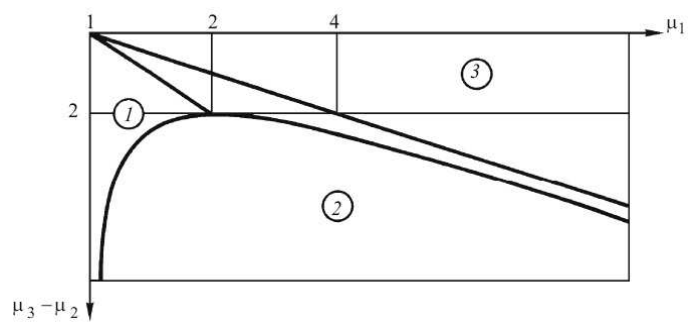

Figure 2: General pattern of trajectories of the system (18) vector fiel near the origin

Proposition 1 Let the inequality (25) holds. Then for $\mu_{3}>\mu_{1}+\mu_{2}\left(\mu_{3}<\mu_{1}+\mu_{2}\right)$ the trivial solution of system (18) is asymptotically stable (is repulsing).

The general pattern of trajectories of the system (18) vector fiel near the origin is represented in Fig. 2 (the domain 1 corresponds the attracting point; the domain 2 corresponds the saddle point; and the domain 3 corresponds the repulsing point).

To ascertain whether a limit cycle can be born near the origin of coordinates, let us analyze the trivial solution of system (18) for stability at the critical relation of the parameters:

$$
\mu_{3}=\mu_{1}+\mu_{2} .
$$

To this end, we change phase variables as $(\alpha, \omega) \mapsto$ $(a, w)$ in system (18):

$$
\begin{gathered}
\alpha=a, \omega=\frac{\left(\mu_{2}+\mu_{1} / 2\right) a-\omega_{0} w}{1+\mu_{1} \mu_{2}+\mu_{2}^{2}}, \\
\omega_{0}=\sqrt{1-\frac{\mu_{1}^{2}}{4}},
\end{gathered}
$$

which leads it to the following system:

$$
\begin{gathered}
a^{\prime}=\left|\omega_{0}\right| w+C_{1} a^{3}+C_{2} a^{2} w+ \\
+C_{3} a w^{2}+\bar{o}_{1}\left(\left(a^{2}+w^{2}\right)^{3 / 2}\right), \\
w^{\prime}=-\left|\omega_{0}\right| a+C_{4} a^{3}+C_{5} a^{2} w+ \\
+C_{6} a w^{2}+C_{7} w^{3}+\bar{o}_{2}\left(\left(a^{2}+w^{2}\right)^{3 / 2}\right),
\end{gathered}
$$

where

$$
\begin{gathered}
C_{1}=\frac{\mu_{2} f_{3}}{6 I n_{0}^{2}}-\frac{H s_{2}}{2 I n_{0}} \frac{\mu_{2}\left(\mu_{2}+\mu_{1} / 2\right)}{1+\mu_{1} \mu_{2}+\mu_{2}^{2}}+\frac{s_{2}}{2 m n_{0}}- \\
-\frac{\mu_{2}}{2}-\frac{\mu_{1}}{12}+ \\
+\frac{\mu_{2}\left(\mu_{1}+\mu_{2}\right)\left(\mu_{2}+\mu_{1} / 2\right)}{2\left(1+\mu_{1} \mu_{2}+\mu_{2}^{2}\right)}+\frac{\mu_{2}\left(\mu_{2}+\mu_{1} / 2\right)^{2}}{\left(1+\mu_{1} \mu_{2}+\mu_{2}^{2}\right)^{2}},
\end{gathered}
$$

$$
\begin{gathered}
C_{2}=\frac{H s_{2}}{2 I n_{0}} \frac{\mu_{2} \omega_{0}}{1+\mu_{1} \mu_{2}+\mu_{2}^{2}}-\frac{2 \mu_{2}\left(\mu_{2}+\mu_{1} / 2\right) \omega_{0}}{\left(1+\mu_{1} \mu_{2}+\mu_{2}^{2}\right)^{2}}- \\
-\frac{\mu_{2}\left(\mu_{2}+\mu_{1} / 2\right) \omega_{0}}{2\left(1+\mu_{1} \mu_{2}+\mu_{2}^{2}\right)}, \\
C_{3}=\frac{\mu_{2} \omega_{0}^{2}}{\left(1+\mu_{1} \mu_{2}+\mu_{2}^{2}\right)^{2}}, \\
C_{4}=-\left(1+\frac{\mu_{1} \mu_{2}}{2}\right) \frac{f_{3}}{6 I n_{0}^{2} \omega_{0}}+ \\
+\frac{H s_{2}}{2 I n_{0}} \frac{\left(\mu_{2}+\mu_{1} / 2\right)\left(1+\mu_{1} \mu_{2} / 2\right)}{\left(1+\mu_{1} \mu_{2}+\mu_{2}^{2}\right) \omega_{0}}+ \\
+\frac{\mu_{2}+\mu_{1} / 2}{2\left(1+\mu_{1} \mu_{2}+\mu_{2}^{2}\right) \omega_{0}} \times \\
\times\left[\left(\mu_{2}+\frac{\mu_{1}}{3}\right)-\frac{\mu_{1} \mu_{2}}{6}\left(\mu_{1}+\mu_{2}\right)\right] \\
C_{5}=-\frac{H s_{2}}{2 I n_{0}} \frac{1+\mu_{1} \mu_{2}-\mu_{1}^{2} / 2}{1+\mu_{1} \mu_{2}+\mu_{2}^{2}}+\frac{s_{2}}{2 m n_{0}}+ \\
+\frac{\mu_{2}\left(\mu_{2}+\mu_{1} / 2\right)^{2}}{\left(1+\mu_{1} \mu_{2}+\mu_{2}^{2}\right)^{2}}+ \\
+\frac{2 \mu_{2}\left(\mu_{1}+\mu_{2}\right)^{2}-4 \mu_{2}-\mu_{1}}{4\left(1+\mu_{1} \mu_{2}+\mu_{2}^{2}\right)},
\end{gathered}
$$$$
C_{6}=-\frac{2 \mu_{2}\left(\mu_{2}+\mu_{1} / 2\right) \omega_{0}}{\left(1+\mu_{1} \mu_{2}+\mu_{2}^{2}\right)^{2}}-\frac{\mu_{2}\left(\mu_{1}+\mu_{2}\right) \omega_{0}}{1+\mu_{1} \mu_{2}+\mu_{2}^{2}},
$$

$C_{7}=\frac{\mu_{2} \omega_{0}^{2}}{\left(1+\mu_{1} \mu_{2}+\mu_{2}^{2}\right)^{2}}, s_{2}=s^{\prime \prime}(0), f_{3}=F^{\prime \prime \prime}(0)$.

We now introduce the following auxiliary index In. More concretely, the index constructed for the system (23) has the form:

$$
\begin{gathered}
\text { In }=6 B_{1}+2 B_{3}+2 B_{5}+6 B_{7}= \\
=\frac{\mu_{2} f_{3}}{I n_{0}^{2}}-\frac{H s_{2}}{I n_{0}} \frac{1+3 \mu_{2}^{2}+5 \mu_{1} \mu_{2} / 2-\mu_{1}^{2} / 2}{1+\mu_{1} \mu_{2}+\mu_{2}^{2}}+4 \frac{s_{2}}{m n_{0}}+ \\
+\frac{\mu_{2}\left(\mu_{1}+\mu_{2}\right)\left(\mu_{2}+2 \mu_{1}\right)+3 \mu_{2}-\mu_{1}}{1+\mu_{1} \mu_{2}+\mu_{2}^{2}}
\end{gathered}
$$

The following proposition gives the necessary and sufficien conditions of asymptotic stability (instability) of the origin for In $\neq 0$.

Proposition 2 If In $<0($ In $>0)$ and the inequality

$$
\left|\mu_{3}-\mu_{2}\right|<2
$$

holds, then the origin of coordinates of the phase plane $\mathbf{R}^{2}\{a, w\}$ of system (23) ((18)) is a weak stable (unstable) focus when the critical parameter relation $\mu_{3}=\mu_{1}+\mu_{2}$ is satisfied. 
The condition (25) is necessary in this case, since the origin of coordinates on the plane $\mathbf{R}^{2}\{a, w\}$ will be a (stable or unstable, strong or weak) focus only if this condition is satisfied

The following theorem is the corollary of wellknown theorem of Poincarè-Andronov-Hopf (see [1, 13]).

Theorem 3 Let inequality (25) holds for system (18). Then:

1) If In $<0$, then for any fixed $\mu_{1}, \mu_{2}$ there exist $\delta_{1}, \delta_{2}>0$, such that the origin of coordinates is a strong stable focus for $\mu_{3} \in\left(\mu_{1}+\mu_{2}, \mu_{1}+\mu_{2}+\delta_{1}\right)$; when $\mu_{3} \in\left(\mu_{1}+\mu_{2}-\delta_{2}, \mu_{1}+\mu_{2}\right)$, the origin is a strong unstable focus surrounded by a stable limit cycle that expands as $\mu_{3}$ decreases from $\mu_{1}+\mu_{2}$ to $\mu_{1}+\mu_{2}-\delta_{2}$ as $\sqrt{\left|\mu_{1}+\mu_{2}-\mu_{3}\right|}$.

2) If In $>0$, then for any fixed $\mu_{1}, \mu_{2}$ there exist $\delta_{1}, \delta_{2}>0$, such that the origin of coordinates is a strong unstable focus for $\mu_{3} \in\left(\mu_{1}+\mu_{2}-\delta_{2}, \mu_{1}+\mu_{2}\right)$; when $\mu_{3} \in\left(\mu_{1}+\mu_{2}, \mu_{1}+\mu_{2}+\delta_{1}\right)$, the origin is a strong stable focus surrounded by an unstable limit cycle that expands as $\mu_{3}$ increases from $\mu_{1}+\mu_{2}$ to $\mu_{1}+\mu_{2}+\delta_{1}$ as $\sqrt{\left|\mu_{1}+\mu_{2}-\mu_{3}\right|}$.

The condition $\mu_{3}>\mu_{1}+\mu_{2}\left(\mu_{3}<\mu_{1}+\mu_{2}\right)$ can easily be tested, in principle, since in each specifi case, these parameters depend either on the firs derivatives with respect to the medium interaction functions $\left(y_{N}, s\right)$ or on their values. And the condition In $<0$ (In $>0$ ), however, is rather difficul to test in each specifi case, since not only the explicit expressions but also highest derivatives, even at separate points, of the medium interaction functions $\left(y_{N}, s\right)$ are unknown for each specifi body.

\section{Data Preparation for Execution of Natural Experiments}

\subsection{Problem of Input of Homogeneous Cir- cular Cylinders in Water}

Further, let return to the problem of input of homogeneous circular cylinders in water (see [2]). The values of physical parameters of cylinders, for which the rectilinear translational deceleration (drag) can be stable in principle, must be related by the relation

$$
\mu_{3}>\mu_{1}+\mu_{2}
$$

(see [4]) or

$$
h \frac{m D^{2}}{I}-2-k \frac{m \sigma D}{I}>0 .
$$

Herewith, if the value in the left-hand side of the inequality (27) is equal to zero then we deal with critical case.

Recall that $D$ is the diameter of the circular cylinder, $\sigma$ is the distance from the center of mass of a body to the front butt end, the constants $I, m$ are the massinertia characteristics of cylinder, the constants $k$ and $h$ are the dimension-free parameters of medium action on the cylinder (see [5]).

For the parameters $k$ and $h$ of the water action on the body with the front circular butt end, the estimates $k=h=0,1$ have already been accepted. Thus the condition (27) allows to try to construct a rigid body (a circular cylinder) for which the rectilinear translational deceleration (drag) could be stable. For this, it takes to choose the parameters $\sigma, D, I, m$ of a cylinder Starting from the condition (27).

Analyzing the inequality (27), we can conclude the following. The inertia-mass parameters of the homogeneous cylinders are such that inequality (27) is not possible to satisfy for $h=0,1$. Indeed, for this value $(h=0,1)$, the left-hand side of (27) is represented in the form

$$
\begin{gathered}
\left.F_{1}(k, h, m, I, \sigma, D)\right|_{k=h=0,1}= \\
=h \frac{m D^{2}}{I}-2-\left.k \frac{m \sigma D}{I}\right|_{k=h=0,1}=F_{2}(\sigma, D) .
\end{gathered}
$$

the right-hand side of which, in turn, always assumes the following negative value (with accuracy up to a positive factor):

$$
-3 D^{2}-12 \sigma D-80 \sigma^{2},
$$

which corresponds to the exponential instability of the rectilinear translational drag; here, we take into account that principal moment of inertia of cylinder is represented in the form

$$
I=m\left(\frac{\sigma^{2}}{3}+\frac{D^{2}}{16}\right) .
$$

Furthermore, if we study the left-hand side of (27) under variation of value $h$ then it can attain zero for the following smallest critical value $h_{*}$ only:

$$
\left(10 h_{*}-\frac{5}{4}\right)-\bar{\sigma}-\frac{20}{3} \bar{\sigma}^{2}=0, \bar{\sigma}=\frac{\sigma}{D},
$$

exceeding the earlier accepted value $h=0,1$ and is equal to

$$
h_{*}=0,125 .
$$

The conditions (31) and (32) allow to draw the following intermediate conclusion. The rectilinear translational deceleration (drag) of the homogeneous 


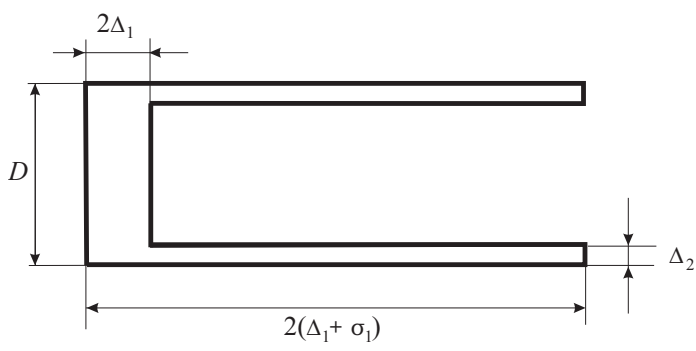

Figure 3: Hollow cylinder ("cartridge")

circular cylinder in the water can not be stable with respect to the perturbations of the angle of attack and angular velocity.

Nevertheless, we note that problem of stability studied can be resolved in accordance with the estimation on this coefficien accepted earlier: $h=0,1$.

\subsection{Problem of Input of Hollow Circular Cylinders in Water}

Let set the problem for determining of geometrical and inertia-mass parameters of the combined rigid body, i.e., the hollow cylinder, for the possible attaining of such a stability. Namely, let imagine a certain hollow cylinder (a "cartridge", Fig. 3), the geometrical and inertia-mass characteristics of which, further, will allow to satisfy the desired inequality for the value $h=0,1$ has been fi ed us.

The combined rigid body studied is represented by the front homogeneous part (a cylinder) of the diameter $D$ and the height $2 \Delta_{1}$ which can be continued by the the lateral partitions of length $2 \sigma_{1}$ and width $\Delta_{2}$ (Fig. 3).

Let calculate the combined body parameters appearing in the inequality (27), i. e., the distance from the center of mass of a body to the front circular butt end $\sigma$, and also the (principal) radius of inertia of a body $\rho$. These values are expressed through the following formulas:

$$
\begin{gathered}
\sigma=\frac{\Delta_{1}^{2} D^{2}+4 \sigma_{1} \Delta_{2}\left(D-\Delta_{2}\right)\left(\sigma_{1}+2 \Delta_{1}\right)}{\Delta_{1}^{2} D^{2}+4 \sigma_{1} \Delta_{2}\left(D-\Delta_{2}\right)}, \\
\rho^{2}=\frac{\Delta_{1}^{2} D^{2}+}{\Delta_{1}^{2} D^{2}+4 \sigma_{1} \Delta_{2}\left(D-\Delta_{2}\right)} \times \\
\times\left\{\frac{4}{3} \Delta_{1}^{2}+\frac{D^{2}}{16}-2 \Delta_{1} \sigma+\sigma^{2}\right\}+ \\
+\frac{4 \sigma_{1} \Delta_{2}\left(D-\Delta_{2}\right)}{\Delta_{1}^{2} D^{2}+4 \sigma_{1} \Delta_{2}\left(D-\Delta_{2}\right)} \times \\
\times\left\{\frac{\sigma_{1}^{2}}{3}+\frac{D^{2}}{8}-\frac{\Delta_{2}\left(D-\Delta_{2}\right)}{4}+\right.
\end{gathered}
$$

$$
\left.+\frac{D^{4} \Delta_{1}^{2}\left(\sigma_{1}+\Delta_{1}\right)^{2}}{\Delta_{1}^{2} D^{2}+4 \sigma_{1} \Delta_{2}\left(D-\Delta_{2}\right)}\right\} .
$$

We can use the complete Eqs. (33), (34), however, it has not solving importances, since it is suffi cient to accept the following admissions:

$$
\Delta_{1}^{2} \approx \Delta_{2}^{2} \approx \Delta_{2} \Delta_{2} \approx 0
$$

Further, we notice that all the geometrical parameters are dimension-free:

$$
\overline{\Delta_{1}}=\frac{\Delta_{1}}{D}, \overline{\Delta_{2}}=\frac{\Delta_{2}}{D}, \overline{\sigma_{1}}=\frac{\sigma_{1}}{D},
$$

furthermore, for simplicity, we omit the bar everywhere.

Then the left-hand side of (27) under the admissions (35) for $h=0,1$ is reduced to the following equality in critical case:

$$
\Delta_{1}\left(-\frac{1}{4}\right)+\sigma_{1} \Delta_{2}\left(\frac{7}{2}\right)-4 \sigma_{1}^{2} \Delta_{2}=0 .
$$

Let fin the critical value $\sigma_{1}^{*}$ of the dimension-free length of the lateral partitions of the combined body. It is equal to

$$
\sigma_{1}^{*}=\frac{7}{16}+\frac{1}{8} \sqrt{\frac{49}{4}-4 \frac{\Delta_{1}}{\Delta_{2}}} .
$$

From Eq. (38), we see that the value $\Delta_{1} / \Delta_{2}$ can oscillate in the following restrictions only:

$$
0<\frac{\Delta_{1}}{\Delta_{2}}<\frac{49}{16}=3,0625
$$

Formally, for $\Delta_{1} \rightarrow 0$ (the front butt end tends to the infinit thin disk) the desired critical value tends to

$$
\sigma_{1}^{*}=0,875
$$

In particular interesting case, for $\Delta_{1}=\Delta_{2}$ it can be found in the form

$$
\sigma_{1}^{*}=\frac{1}{16}(7+\sqrt{33}) \approx 0,797,
$$

and also for $\Delta_{1} / \Delta_{2} \rightarrow 49 / 16$ the desired critical value tends to

$$
\sigma_{1}^{*}=0,4375 \text {. }
$$

Thus, we can chose the value $\sigma_{1}^{*}$ in the restrictions

$$
0.4375 \leq \sigma_{1}^{*} \leq 0.875
$$

in spite of the fact that the expressions (40)-(42) deal with the suitable cases only. 
Also, in particular, if we accept $\Delta_{1}=\Delta_{2}=0,1$ (i. e., if $D=30 \mathrm{~mm}$, then $\Delta_{1}=\Delta_{2}=3 \mathrm{~mm}$ ); then the dimensional length of the lateral partitions can be equal to $2 \sigma_{1} \approx 1,6 D \approx 47,8 \mathrm{~mm}$, and the complete critical length of all the combined body is equal to $47,8+6 \approx 54 \mathrm{~mm}$.

In conclusion we shall notice that if it needs to "correct" the constant value $h$ of a medium action on a body for the experiment execution, that the desired expression for the value $\sigma_{1}^{*}$ will be represented as follows. The linearized critical equality (37) will be rewritten in the form

$$
\Delta_{1}\left(10 h-\frac{5}{4}\right)+\sigma_{1} \Delta_{2}\left(40 h-\frac{1}{2}\right)-4 \sigma_{1}^{2} \Delta_{2}=0,
$$

and the desired value $\sigma_{1}^{*}$ can be found from the equality

$$
\begin{gathered}
\sigma_{1}^{*}=\frac{1}{8}\left\{\left(40 h-\frac{1}{2}\right)+\right. \\
+\sqrt{\left.\left(40 h-\frac{1}{2}\right)^{2}+16\left(10 h-\frac{5}{4}\right) \frac{\Delta_{1}}{\Delta_{2}}\right\}} .
\end{gathered}
$$

\subsection{Possibilities of a Rigid Body Motion in a Resisting Medium with Restricted An- gles of Attack}

As was mentioned in previous sections, if the parameters of the problem allow the existence of critical case (the left-hand side of the Eq. (27) is equal to zero), then the rectilinear translational deceleration (drag) of a body can be stable as well as unstable with respect to the perturbations of the angle of attack and angular velocity, in depending on the higher derivatives of the medium action functions $y_{N}$ and $s$.

There were found the sufficien conditions for such stability as well as instability (see above), including the inequalities with the higher derivatives of the medium action functions. But the main difficult is because of it is not possible to measure these derivatives in the explicit form in the experiment.

Let demonstrate how does it make possible to study the behavior of a body nearby the rectilinear translational deceleration (i.e., the stable as well as instable angular oscillations) using the experimental information, hereunder, estimating not explicitly the higher derivatives of the medium action functions.

At the beginning we note that the following inequality guaranteing the oscillate stability as well as instability, holds at least in the case of the products which are offered above (it makes possible to variate the mass of a body making the products from the met- als of the different density):

$$
\frac{D I \rho_{0}}{m^{2}}<\frac{8 k}{c_{x} \pi},
$$

where we add the following parameters to the known ones: $\rho_{0}$ is the density of the flui (it is the water in this case), $c_{x}=0,82$ is the dimension-free coefficien of frontal resistance.

Indeed, in the system SGS the inequality (46) is equivalent to $D \rho^{2} / m<0,31,[m]=g,[D]=[\rho]=$ $s m$, where $\rho$ is the (principal) radius of inertia expressing by the formula (34).

Further, it is necessary to obtain the information about not less than three semi-oscillations (i.e., one and half periods of the oscillations) with the amplitudes $a_{1}, a_{2}, a_{3}$ during the experiment execution in the case of oscillated character of the motion. Using the parameter values which are close to the critical case, we have two conclusions on the stability of the key regime.

For the beginning we give an important remark on the consequent changes of the values of amplitudes $a_{1}, a_{2}, a_{3}$ measuring in the experiment.

Remark 4 The sequence of the ratio of the values $a_{1}, a_{2}, a_{3}, \ldots$

$$
\frac{a_{2}}{a_{1}}, \frac{a_{3}}{a_{2}}, \ldots
$$

(hereinafter, if it is possible to measure not three semi-oscillations but one more ones) is able to determine the character of the oscillate process in majority. Also, if the values

$$
a_{1}, a_{2}, a_{3}, \ldots
$$

look like the increasing (decreasing) geometric progression (in particular, the relations $a_{2} / a_{1}, a_{3} / a_{2}$ are equal approximately), then we can confirm the sufficiently quick growing (fading) of the angular oscillations with share of confidence. And if the values (48) increase, and its ratio $a_{2} / a_{1}, a_{3} / a_{2}, \ldots$ (see Eq. (47)) decrease explicitly, then we should say about possible transition to the angular oscillations of the bounded amplitude.

I. Let we observe the stable oscillations with respect to the angle of deviation during the natural experiment execution with the parameters corresponding to the critical case. Then, under the small decreasing of the body longitudal length (see a sample 1 below), one can appear the small fading of the angular oscillations (Fig. 4).

Under the small increasing of the body longitudal length (see a sample 2 below), we should wait the increasing of the angular oscillations, and, in sequel, can 


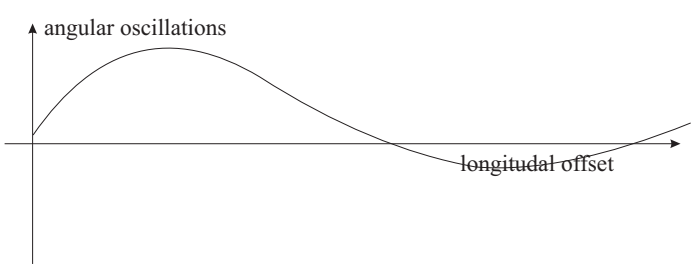

Figure 4:

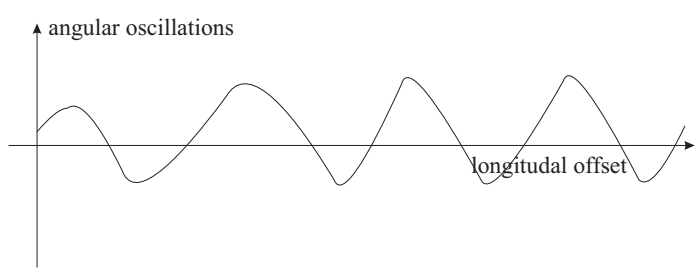

Figure 5:

observe the stable auto-oscillations of the body (Fig. 5). Herewith, it takes to pay attention on the amplitude variation velocity of the oscillations (see Remark 4).

Since the cases under consideration correspond to a weak focus, a more accurate mechanical interpretation is significantl difficult

Furthermore, executing the experiment again for the product from the sample 2 for sufficientl big perturbations of the initial angle of attack and (or) angular velocity, it makes possible the transition to the stable angular self-oscillations with the finit amplitudes (Fig. 6) which are similar to the previous case in Fig. 5.

Sample 1: the longitudal length of a body is equal to $50<54(\mathrm{~mm})$.

Sample 2: the longitudal length of a body is equal to $60>54(\mathrm{~mm})$.

II. Let we observe the growth of the angular stable oscillations during the natural experiment execution with the parameters corresponding to the critical case. Then, under the small decreasing of the body longitudal length (see a sample 1 below), we can also observe the stable oscillations of the bounded amplitude (i. e., the transition from the unstable angular auto-oscillations of the body, Fig. 7). Herewith, it takes to pay attention on the amplitude variation velocity of the oscillations (see Remark 4).

Furthermore, executing the experiment again for the product from the sample 1 for the finit perturbations of the initial angle of attack and (or) angular velocity, it makes possible the transition from the unstable angular self-oscillations to its growth (Fig. 8 ). And for the small increasing of the body longitu-

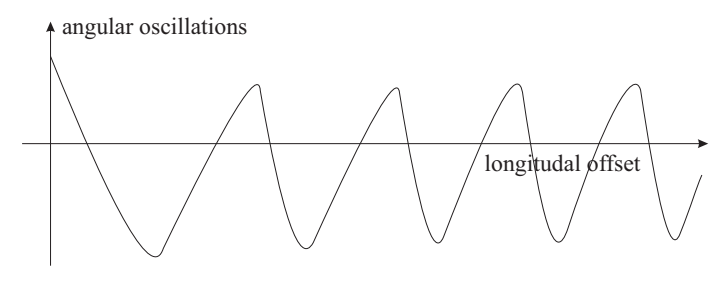

Figure 6:

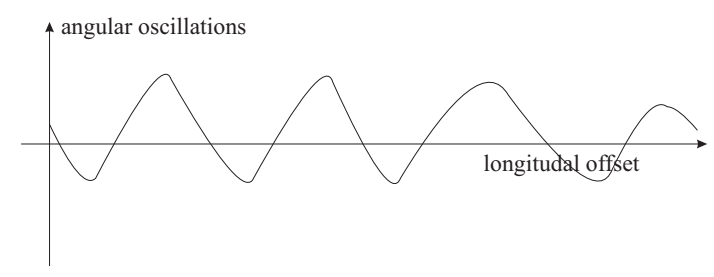

Figure 7:

dal length (see a sample 2 above) we should wait the growth of the angular oscillations (Fig. 9).

\section{Conclusions}

Under the studying of model considered, we fin the sufficien conditions of the asymptotic stability of one of the key regime (rectilinear translational deceleration). In application to the homogeneous circular cylinders, we represent the concrete estimations on its inertia-mass characteristics, herewith, we take into account the results of the experiments executing earlier, including the results in obtaining of the dimensionfree parameters of the water action on the cylinders.

In this work, we also show that under the certain conditions on the higher derivatives of the medium action functions (the arm of the action force and the coefficien of resistance) it makes possible the presence in the system the stable as well as unstable autooscillating regimes of motion. Therefore, the measurement of higher derivatives of these medium action functions is the resistless difficult, since for every concrete the body, not only the explicit form, but even the signs of higher derivatives are not known in the separate points of such functions.

Under the process of application of the methods (which are obtained earlier) of studying of dissipative dynamic systems of the certain forms arising in the problem on free deceleration, we obtain the new multi-parameter family of the phase patterns on two-dimensional cylinders of the quasi-velocities; such family consists of the infinit set of topologically nonequivalent patterns changing its topological type by the degenerate way under the variation of the sys- 


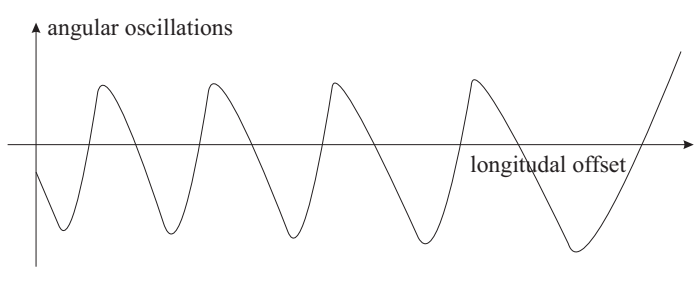

Figure 8:

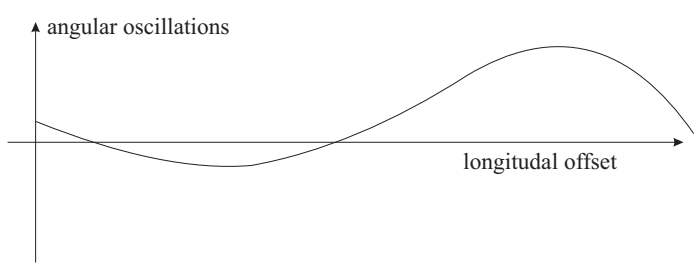

Figure 9:

tem parameters. This family obtained possesses the stable as well as unstable self-oscillatory regimes in the finit range of the angle of attack. Herewith, the domain of the physical parameters is the set of the fi nite measure in all infinite-measure space of the system parameters, therefore, the patterns obtained are the typical.

The obtained results allow to construct the hollow circular cylinders (i. e., "the cartridge case"), the use of which can provide the necessary stability under the execution of the additional natural experiments (see also $[14,15])$.

The approach considered in this paper, in principle, can also be combined with well-known methods of computer modeling $[16,17]$.

Acknowledgements: This work was supported by the Russian Foundation for Basic Research, project no. 19-01-00016.

\section{References:}

[1] Shamolin M. V. Some questions of the qualitative theory of ordinary differential equations and dynamics of a rigid body interacting with a medium, J. Math. Sci., 2002, 110, no. 2, pp. 2526-2555.

[2] Shamolin M. V. New integrable cases and families of portraits in the plane and spatial dynamics of a rigid body interacting with a medium, J. Math. Sci., 2003, 114, no. 1, pp. 919-975.

[3] Shamolin M. V. Foundations of differential and topological diagnostics, J. Math. Sci., 2003, 114, no. 1, pp. 976-1024.
[4] Shamolin M. V. Classes of variable dissipation systems with nonzero mean in the dynamics of a rigid body, J. Math. Sci., 2004, 122, no. 1, pp. 2841-2915.

[5] Shamolin M. V. On integrability in elementary functions of certain classes of nonconservative dynamical systems, J. Math. Sci., 2009, 161, no. 5, pp. 734-778.

[6] Shamolin M. V. Dynamical systems with variable dissipation: approaches, methods, and applications, J. Math. Sci., 2009, 162, no. 6, pp. 741-908.

[7] Shamolin M. V. Classificatio of complete integrability cases in four-dimensional symmetric rigid-body dynamics in a nonconservative field J. Math. Sci., 2010, 165, no. 6, pp. 743754.

[8] Trofim v V. V., and Shamolin M. V. Geometric and dynamical invariants of integrable Hamiltonian and dissipative systems, J. Math. Sci., 2012, 180, no. 4, pp. 365-530.

[9] Shamolin M. V. Comparison of complete integrability cases in Dynamics of a two-, three-, and four-dimensional rigid body in a nonconservative field J. Math. Sci., 2012, 187, no. 3, pp. 346-459.

[10] Shamolin M. V. Some questions of qualitative theory in dynamics of systems with the variable dissipation, J. Math. Sci., 2013, 189, no. 2, pp. 314-323.

[11] Shamolin M. V. Variety of Integrable Cases in Dynamics of Low- and Multi-Dimensional Rigid Bodies in Nonconservative Force Fields, J. Math. Sci., 2015, 204, no. 4, pp. 479-530.

[12] Shamolin M. V. Classificatio of Integrable Cases in the Dynamics of a Four-Dimensional Rigid Body in a Nonconservative Field in the Presence of a Tracking Force, J. Math. Sci., 2015, 204, no. 6, pp. 808-870.

[13] Shamolin M. V. Some Classes of Integrable Problems in Spatial Dynamics of a Rigid Body in a Nonconservative Force Field, J. Math. Sci., 2015, 210, no. 3, pp. 292-330.

[14] Shamolin M. V. New Cases of Integrable Fifth-Order Systems with Dissipation, Doklady Physics, 2019, 64, no. 4, pp. 189-192.

[15] Shamolin M. V. New Cases of Integrable Seventh-Order Systems with Dissipation, Doklady Physics, 2019, 64, no. 8, pp. 330-334.

[16] Banks, J., Carson, J.S., Nelson, B.L. and Nicol, D.M. Discrete-Event System Simulation. 5th Edition, Prentice Hall, Upper Saddle River, 2009. 
[17] Adriano Torri, Oscar Tamburis, Teresa Abbate, Alessandro Pepino, New Perspectives for Workfl w Analysis in the Health Italian Sector through Discrete Event Simulation: The Case of a Department of Laboratory Medicine, Intelligent Information Management, 2015, 7, no. 3 .
Creative Commons Attribution License 4.0 (Attribution 4.0 International, CC BY 4.0)

This article is published under the terms of the Creative Commons Attribution License 4.0

https://creativecommons.org/licenses/by/4.0/deed.en US 